\title{
Filgrastim induced different mechanical responses in guinea pig and rat hearts
}

\author{
Cecilia Villa Etchegoyen", Guillermo A Keller², Sebastian Mrad', Roberto A Diez¹, Guillermo Di \\ Girolamo ${ }^{1}$, Patricia Bonazzola ${ }^{3}$
}

${ }^{1}$ Laboratory of Cardiovascular Surveillance of Drugs - II Chair of Pharmacology - School of Medice - Buenos Aires University, Argentina, ${ }^{2}$ Drug Surveillance \& Safety Center, School of Medicine, Universidad de Buenos Aires, Buenos Aires, Argentina, ${ }^{3}$ Laboratory of cardiac metabolism. Cardiovascular Research Institute (ININCA), Buenos Aires Univerity-National Council for Scientific and Technical Research (CONICET), Argentina

Introduction: Previous studies in adult rat heart showed a negative inotropic effect with the human Granulocyte Colony Stimulator Factor (hGCS-F), filgrastrim $(800 \mathrm{ng} / \mathrm{ml})$. Due to electrophysiological studies on guinea pig are necessary in cardiovascular surveillance of drugs (ICH S7B), in a first stage, the mechanical behaviour of the heart during perfusion of filgrastim was studied in this species.

Methods:Using Langendorff technique, hearts from 13 Ssi:AL guinea pigs and 9 Wistar rats were isolated and arterially perfused. The left ventricular developed pressure (P) was constantly recorded through a latex balloon placed into the left ventricle and electrically paced at $3.5 \mathrm{~Hz}$. After $50 \mathrm{~min}$-stabilization, hearts were exposed to increasing and cumulatively doses of filgrastim $(10-30-100-400-800 \mathrm{ng} / \mathrm{ml}), 10 \mathrm{~min}$ each. $\mathrm{P}$ is expressed as percentage of pre-treatment value. An indirect measurement of the sarcoplasmic reticulum (SR) calcium content was assessed through caffeine (20mM)-low sodium $(36 \mathrm{mM})$ induced contracture during 20 minutes and the associated area under the curve of contracture (AUCC). Results are expressed as mean-SEM.

Results: In guinea pig, a significant increase in $\mathrm{P}$ at $800 \mathrm{ng} / \mathrm{ml}$ of filgrastim was observed (126.4-9.3\%(n=8) vs. 92.4-2.1\% $(n=5), p=0.01)$. No significant differences were evidenced on both, caffeine-low sodium induced contracture and the AUCC. This positive inotropic effect (IE) is independent of SR-calcium content suggesting that it could be related either with changes in sarcolemmal calcium availability or a direct effect on contractile machinery.

Instead, in adult rat hearts, the drop of $\mathrm{P}$ in the presence of filgrastim (85.6-4.7\% ( $\mathrm{n}=4)$ vs. $98.3-1.7 \%(\mathrm{n}=5)$, $\mathrm{p}=0.01)$ was accompanied by an increase on both, contracture level and AUCC (1302-69 mmHg.min vs. 886-176 mmHg.min, $\mathrm{p}=0.04$ ) suggesting that the observed IE could be related with a diminished calcium availability due to an inhibition of calcium release from SR.

Conclusion: The opposed drug behaviour could be explained by the known differences in calcium handling between both species and denote the importance of the animal model used. As functional calcium handling and structural properties in guinea pig are closer to human heart make this animal model a better one for evaluation of mechanical and electrophysiological behaviour in the context of cardiac safety profile of drugs. 